\title{
Accurate Evaluation of Segment-level Machine Translation Metrics
}

\author{
Yvette Graham $^{\dagger \ddagger} \quad$ Nitika Mathur ${ }^{\dagger} \quad$ Timothy Baldwin $^{\dagger}$ \\ ${ }^{\dagger}$ Department of Computing and Information Systems, The University of Melbourne \\ ${ }^{\ddagger}$ ADAPT Research Centre, Trinity College Dublin \\ ygrahamescss.tcd.ie, nmathurestudent.unimelb.edu.au, tbeldwin.net
}

\begin{abstract}
Evaluation of segment-level machine translation metrics is currently hampered by: (1) low inter-annotator agreement levels in human assessments; (2) lack of an effective mechanism for evaluation of translations of equal quality; and (3) lack of methods of significance testing improvements over a baseline. In this paper, we provide solutions to each of these challenges and outline a new human evaluation methodology aimed specifically at assessment of segment-level metrics. We replicate the human evaluation component of WMT-13 and reveal that the current state-of-the-art performance of segment-level metrics is better than previously believed. Three segment-level metrics - METEOR, NLEPOR and SENTBLEUMOSES - are found to correlate with human assessment at a level not significantly outperformed by any other metric in both the individual language pair assessment for Spanish-toEnglish and the aggregated set of 9 language pairs.
\end{abstract}

\section{Introduction}

Automatic segment-level machine translation (MT) metrics have the potential to greatly advance MT by providing more fine-grained error analysis, increasing efficiency of system tuning methods and leveraging techniques for system hybridization. However, a major obstacle currently hindering the development of segment-level metrics is their evaluation. Human assessment is the gold standard against which metrics must be evaluated, but when it comes to the task of evaluating translation quality, human annotators are notoriously inconsistent. For example, the main venue for evaluation of metrics, the annual Workshop on Statistical Machine Translation (WMT), reports disturbingly low inter-annotator agreement levels and highlights the need for better human assessment of MT. WMT-13, for example, report Kappa coefficients ranging from 0.075 to 0.324 for assessors from crowd-sourcing services, only increasing to between 0.315 and 0.457 for MT researchers (Bojar et al., 2013a). For evaluation of metrics that operate at the system or document-level such as BLEU, inconsistency in individual human judgments can, to some degree, be overcome by aggregation of individual human assessments over the segments within a document. However, for evaluation of segment-level metrics, there is no escaping the need to boost the consistency of human annotation of individual segments.

This motivates our analysis of current methods of human evaluation of segment-level metrics, and proposal of an alternative annotation mechanism. We examine the accuracy of segment scores collected with our proposed method by replicating components of the WMT-13 human evaluation (Bojar et al., 2013b), with the sole aim of optimizing agreement in segment scores to provide an effective gold standard for evaluating segment-level metrics. Our method also supports the use of significance testing of segment-level metrics, and tests applied to the WMT-13 metrics over nine language pairs reveal for the first time which segment-level metrics outperform others. We have made available code for acquiring accurate segment-level MT human evaluations from the crowd, in addition to significance 
testing competing segment-level metrics, at:

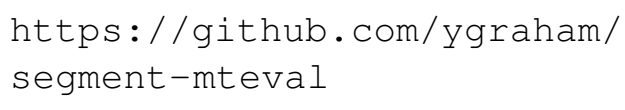

\section{WMT-style Evaluation of Segment-level MT Metrics}

Since 2008, the WMT workshop series has included a shared task for automatic metrics, and as with the translation shared task, human evaluation remains the official gold standard for evaluation. In order to minimize the amount of annotation work and enforce consistency between the primary shared tasks in WMT, the same evaluations are used to evaluate MT systems in the shared translation task, as well as MT evaluation metrics in the document-level metrics and segment-level metrics tasks. Although WMT have trialled several methods of human evaluation over the years, the prevailing method takes the form of ranking a set of five competing translations for a single source language (SL) input segment from best to worst. A total of ten pairwise human relative preference judgments can be extracted from each set of five translations. Performance of a segment-level metric is assessed by the degree to which it corresponds with human judgment, measured by the number of metric scores for pairs of translations that are either concordant (Con) or discordant (Dis) with those of a human assessor, which the organizers describe as "Kendall's $\tau$ ":

$$
\tau=\frac{\mid \text { Con }|-| \text { Dis } \mid}{\mid \text { Con }|+| \text { Dis } \mid}
$$

Pairs of translations deemed equally good by a human assessor are omitted from evaluation of segment-level metrics (Bojar et al., 2014).

There is a mismatch between the human judgments data used to evaluate segment-level metrics and the standard conditions under which Kendall's $\tau$ is applied, however: Kendall's $\tau$ is used to measure the association between a set of observations of a single pair of joint random variables, $X$ (e.g. the human rank of a translation) and $Y$ (e.g. the metric score for the same translation). A conventional application of Kendall's $\tau$ would be comparison of all pairs of values within $X$ with each corresponding pair within $Y$. Since the human assessment data is, however, a large number of separately ranked sets of five competing translations and not a single ranking of all translations, it is not possible to compute a single Kendall's $\tau$ correlation. ${ }^{1}$ The formula used to assess the performance of a metric in the task, therefore, is not what is ordinarily understood to be a Kendall's $\tau$ coefficient, but, in fact, equivalent to a weighted average of all Kendall's $\tau$ for each humanranked set of five translations.

A more significant problem, however, lies in the inconsistency of human relative preference judgments within data sets. Since overall scores for metrics are described as correlations, possible values achievable by any metric could be expected to lie in the range $[-1,1]$ (or " \pm 1 "). This is not the case, and achievements of metrics are obscured by contradictory human judgments. Before any metric has provided scores for segments, for example, the maximum and minimum correlation achievable by a participating metric can be computed as, in the case of WMT-13:

- Russian-to-English: \pm 0.92

- Spanish-to-English: \pm 0.90

- French-to-English: \pm 0.90

- German-to-English: \pm 0.92

- Czech-to-English: \pm 0.89

- English-to-Russian: \pm 0.90

- English-to-Spanish: \pm 0.90

- English-to-French: \pm 0.91

- English-to-German: \pm 0.90

- English-to-Czech: \pm 0.87

If we are interested in the relative performance of metrics and take a closer look at the formula used to contribute a score to metrics, we can effectively ignore the denominator $(|\operatorname{Con}|+\mid$ Dis $\mid)$, as it is constant for all metrics. The numerator $(\mid$ Con $|-|$ Dis $\mid)$ is what determines our evaluation of the relative performance of metrics, and although the formula appears to be a straightforward subtraction of counts of concordant and discordant pairs, due to the large numbers of contradictory human relative preference judgments in data sets, what this number actually represents is not immediately obvious. If, for example, translations $A$ and $B$ were scored by a metric such that metric_score $(A)>$ metric_score $(B)$, one

\footnotetext{
${ }^{1}$ This would in fact require all $(\mid$ MT systems $|\times|$ distinct segments $\mid$ ) translations included in the evaluation to be placed in a single rank order.
} 
might expect an addition or subtraction of 1 depending on whether or not the metric's scores agreed with those of a human. Instead, however, the following is added:

$$
\begin{aligned}
& (\max (|A>B|,|A<B|) \\
& \quad-\min (|A>B|,|A<B|)) \times d
\end{aligned}
$$

where:

$$
\begin{aligned}
&|A>B|= \text { \# human judgments where } \mathrm{A} \text { was } \\
& \text { preferred over } \mathrm{B} \\
&|A<B|= \text { \# human judgments where } \mathrm{B} \text { was } \\
& \text { preferred over } \mathrm{A} \\
& d=\left\{\begin{aligned}
1 & \text { if }|A<B|>|A>B| \\
-1 & \text { if }|A<B|<|A>B|
\end{aligned}\right.
\end{aligned}
$$

For example, translations of segment 971 for Czechto-English systems uedin-heafield and uedin-wmt13 were compared by human assessors a total of 12 times: the first system was judged to be best 4 times, the second system was judged to be best 2 times, and the two systems were judged to be equal 6 times. This results in a score of $4-2$ for a system-level metric that scores the uedin-heafield translation higher than uedin-wmt13 (tied judgments are omitted), or score of $2-4$ in the converse case.

Another challenge is how to deal with relative preference judgments where two translations are deemed equal quality (as opposed to strictly better or worse). In the current setup, tied translation pairs are excluded from the data, meaning that the ability for evaluation metrics to evaluate similar translations is not directly evaluated, and a metric that manages to score two equal quality translations closer, does not receive credit. A segment-level metric that can accurately predict not just disparities between translations but also similarities is likely to have high utility for MT system optimization, and is possibly the strongest motivation for developing segment-level metrics in the first place. In WMT-13, however, 24\% of all relative preference judgments were omitted on the basis of ties, broken down as follows:

- Spanish-to-English: $28 \%$

- French-to-English: $26 \%$

- German-to-English: 27\%

- Czech-to-English: $25 \%$

- Russian-to-English: 24\%
- English-to-Spanish: $23 \%$

- English-to-French: 23\%

- English-to-German: 20\%

- English-to-Czech: 16\%

- English-to-Russian: 27\%

Although significance tests for evaluation of MT systems and document-level metrics have been identified (Koehn, 2004; Graham and Baldwin, 2014; Graham et al., 2014b), no such test has been proposed for segment-level metrics, and it is unfortunately common to conclude success without taking into account the fact that an increase in correlation can occur simply by chance. In the rare cases where significance tests have been applied, tests or confidence intervals for individual correlations form the basis for drawing conclusions (Aziz et al., 2012; Machacek and Bojar, 2014). However, such tests do not provide insight into whether or not a metric outperforms another, as all that's required for rejection of the null hypothesis with such a test is a likelihood that an individual metric's correlation with human judgment is not equal to zero. In addition, data sets for evaluation in both document and segment-level metrics are not independent and the correlation that exists between pairs of metrics should also be taken into account by significance tests.

\section{Segment-Level Human Evaluation}

Many human evaluation methodologies attempt to elicit precisely the same quality judgment for individual translations from all assessors, and inevitably produce large numbers of conflicting assessments in the process, including from the same individual human judge (Callison-Burch et al., 2007; CallisonBurch et al., 2008; Callison-Burch et al., 2009). An alternative approach is to take into account the fact that different judges may genuinely disagree, and allow assessments provided by individuals to each contribute to an overall estimate of the quality of a given translation.

In an ideal world in which we had access to assessments provided by the entire population of qualified human assessors, for example, the mean of those assessments would provide a statistic that, in theory at least, would provide a meaningful segment-level human score for translations. If it were possible to collect assessments from the entire 
population we could directly compute the true mean score for a translation segment. This is of course not possible, but thanks to the law of large numbers we can make the following assumption:

Given a sufficiently large assessment sample for a given translation, the mean of assessments will provide a very good estimate of the true mean score of the translation sourced from the entire assessor population.

What the law of large numbers does not tell us, however, is, for our particular case of translation quality assessment, precisely how large the sample of assessments needs to be, so that the mean of scores provides a close enough estimate to the true mean score for any translation. For a sample mean for which the variance is known, the required sample size can be computed for a specified standard error. However, due to the large number of distinct translations we deal with, the variance in sample score distributions may change considerably from one translation to the next. In addition, the choice as to what exactly is an acceptable standard error in sample means would be somewhat arbitrary. On the one hand, if we specify a standard error that's lower than is required, and subsequently collect more repeat assessments than is needed, we would be wasting resources that could, for example, be targeted at the annotation of additional translation segments.

Our solution is to empirically investigate the impact on sample size of repeat assessments on the mean score for a given segment, and base our determination of sample size on the findings. Since we later motivate the use of Pearson's correlation to measure the linear association between human and metric scores (see Section 4), we base our investigation on Pearson's correlation.

We collect multiple assessments per segment to create score distributions for segments for a fixed set per language pair. This is repeated twice over the same set of segments to generate two distinct sets of annotations: one set is used to estimate the true mean score, and the second set is randomly downsampled to simulate a set of assessments of fixed sample size. We measure the Pearson correlation between the true mean score and different numbers of

\begin{tabular}{ccc}
\hline $\begin{array}{c}\text { Language } \\
\text { pair }\end{array}$ & \# translations & $\begin{array}{c}\text { \# assessments } \\
\text { per translation }\end{array}$ \\
\hline es-en & 280 & 40 \\
en-es & 140 & 19 \\
en-ru & 140 & 15 \\
en-de & 140 & 14 \\
\hline
\end{tabular}

Table 1: Datasets used to assess translation assessment sample size

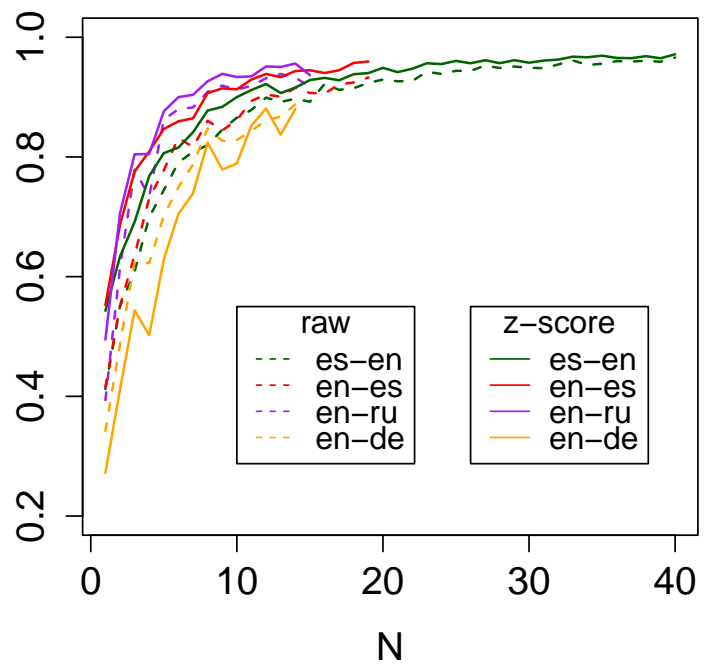

Figure 1: Correlation $(r)$ of translation quality estimates between the initial and repeat experiment runs for each of the four language pairs from WMT-13, for sample size $N$ and based on raw and standardized $(z)$ scores.

assessments for a given assessment, to ask the question: how many assessments must be collected for a given segment to obtain mean segment scores that truly reflects translation quality? Scores are sampled according to annotation time to simulate a realistic setting.

\subsection{Translation Assessment Sample Size}

MTurk was used to collect large numbers of translation assessments, in sets of 100 translations per assessment task (or "HIT" in MTurk parlance). The HITS were structured to include degraded translations and repeat translations, and rated on a continuous Likert scale with a single translation assessment displayed to the assessor at one time (Graham et al., 2014a; Graham et al., 2013). This supports accurate quality-control as well as normalisation of translation scores for each assessor. The assessment 

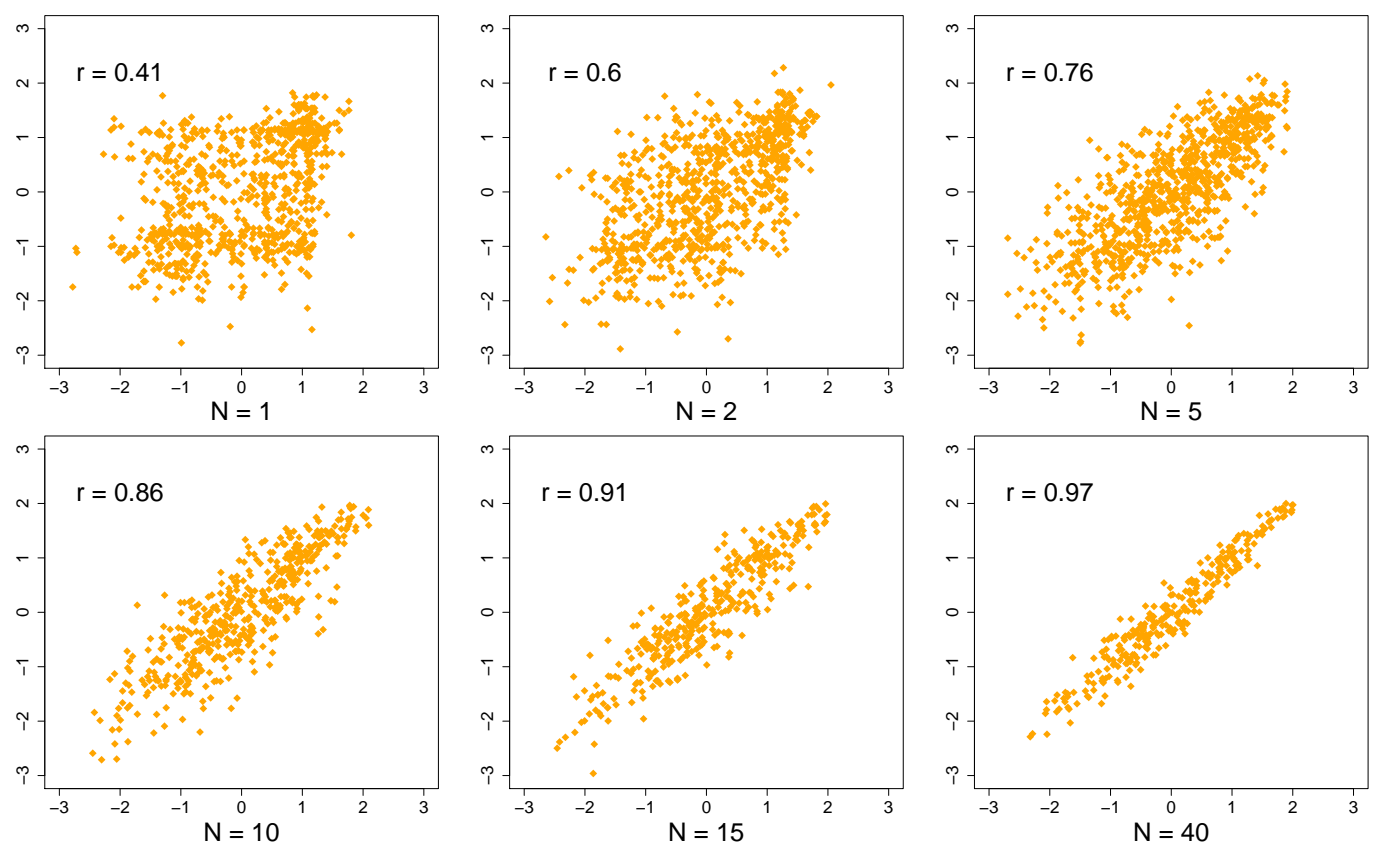

Figure 2: Plots and correlation $(r)$ of translation quality assessments in the initial ( $x$-axis) and replicate experiments $(y$ axis) for Spanish-to-English over WMT-13, where each point represents a standardized segment-level score computed as the mean of the $N$ individual assessments for that plot.

task was posed as a monolingual task, where assessors were asked to rate the degree to which the MT system output adequately expressed the meaning of the corresponding reference translation. Translations were sampled at random from the WMT-13 data sets for the four language pairs, as detailed in Table 1. Due to low-quality assessors on MTurk and the need for assessments solely for quality assurance purposes, the exercise required a substantial number of individual assessments. For Spanish-toEnglish, for example, a total of (280 translations + 120 translations for quality-control purposes) $\times 40$ assessments per translation $\times 2$ separate data collections $\times \sim 2$ to allow for filtering of low-quality assessors $=\sim 64 \mathrm{k}$ assessments were collected; after quality control filtering and removing the qualitycontrol translations, around $22 \mathrm{k}$ assessments were used for the actual experiment.

Figure 1 shows the Pearson correlation between mean segment-level scores calculated for varying numbers of assessments $(N)$, and the full set of assessments for the second set of assessments. For each language pair, we calculate the correlation first over the raw segment scores and second over standardized scores, based on the method of Graham et al. (2014a). ${ }^{2}$ For all language pairs, although the correlation is relatively low for single assessments, as the sample size increases, it increases, and by approximately $N=15$ assessments, for all four language pairs, the correlation reaches $r=0.9$. For Spanish-to-English, for which most assessments were collected, when we increase the number of assessments to $N=40$ per translation, the correlation reaches $r=0.97$. Figure 2 is a set of scatter plots for mean segment-level scores for Spanish-toEnglish rising, for varying sample sizes $N$.

As expected, the larger the sample size of assessments, the greater the agreement with the true mean score, but what is more surprising is that with as few as 15 assessments, the scores collected in the two separate experiments correlate extremely well, and provide what we believe to be a sufficient stability to evaluate segment-level metrics.

\footnotetext{
${ }^{2}$ Standardized segment scores are computed by standardizing individual raw scores according to the mean and standard deviation of individual assessors, and then combined into mean segment scores.
} 


\section{Segment-level Metric Evaluation}

Since the scores generated by our method are continuous and segment-level metrics are also required to output continuous-valued scores, we can now compare the scores directly using Pearson's correlation. Pearson's correlation has three main advantages for this purpose. Firstly, the measure is unit-free, so metrics do not have to produce scores on the same scale as the human assessments. Secondly, scores are absolute as opposed to relative and therefore more intuitive and ultimately more powerful; for example, we are able to evaluate metrics over the $20 \%$ of translations of highest or lowest quality in the test set. Finally, the use of Pearson's correlation facilitates the measurement of statistical significance in correlation differences.

It is important to point out, however, that moving from Kendall's $\tau$ over relative preference judgments to Pearson's $r$ over absolute scores does, in fact, change the task required of metrics in one respect: previously, there was no direct evaluation of the scores generated by a metric, nor indeed did the evaluation ever directly compare translations for different source language inputs (as relative preference judgments were always relative to other translations for the same input). Pearson's correlation, on the other hand, compares scores across the entire test set.

\subsection{Significance Testing of Segment-level Metrics}

With the move to Pearson's correlation, we can also test statistical significance in differences between metrics, based on the Williams test (Williams, 1959), ${ }^{3}$ which evaluates significance in a difference in dependent correlations (Steiger, 1980). As suggested by Graham and Baldwin (2014), the test is appropriate for evaluation of document-level MT metrics since the data is not independent, and for similar reasons, the test can also be used for evaluation of segment-level metrics.

\subsection{Spanish-to-English Segment-level Metrics}

We first carry out tests for Spanish-to-English segment-level metrics from WMT-13. In our experiments in Section 3.1, we used only a sub-sample

\footnotetext{
${ }^{3}$ Also sometimes referred to as the Hotelling-Williams test.
}

\begin{tabular}{lcc}
\hline Metric & $r$ & $\tau$ \\
\hline METEOR & 0.484 & 0.324 \\
NLEPOR & 0.483 & 0.281 \\
SENTBLEU-MOSES & 0.465 & 0.266 \\
DEP-REF-EX & 0.453 & 0.307 \\
DEP-REF-A & 0.453 & 0.312 \\
SIMPBLEUP & 0.450 & 0.287 \\
SIMPBLEUR & 0.444 & 0.388 \\
LEPOR & 0.408 & 0.236 \\
UMEANT & 0.353 & 0.202 \\
MEANT & 0.342 & 0.202 \\
TERRORCAT & 0.313 & 0.313 \\
\hline
\end{tabular}

Table 2: Pearson's correlation and Kendall's $\tau$ between WMT-13 segment-level metrics and human assessment for Spanish-to-English (ES-EN). Note that Kendall's $\tau$ is based on the WMT-13 formulation, and the preference judgments from WMT-13.

of segments, so the first thing is to collect assessments for the remaining Spanish-to-English translation segments using MTurk, based on a sample of at least 15 assessments. A total of 24 HITs of 100 translations each were posted on MTurk; after removal of low quality workers $(\sim 50 \%)$ and quality control items (a further 30\%), this resulted in 840 translation segments with 15 or more assessments each. The scores were standardized and combined into mean segment scores.

Table 2 shows the Pearson's correlation for each metric that participated in the WMT-13 Spanish-toEnglish evaluation task, along with the Kendall's $\tau$ based on the original WMT-13 methodology and relative preference assessments. Overall, when we compare correlations using the new evaluation methodology to those from the original evaluation, even though we have raised the bar by assessing the raw numeric outputs rather than translating them into preference judgments relative to other translations for the same SL input, all metrics achieve higher correlation with human judgment than reported in the original evaluation. This indicates that the new evaluation setup is by no means unrealistically difficult, and that even though it was not required of the metrics in the original task setup, the metrics are doing a relatively good job of absolute scoring of translation adequacy. In addition, 


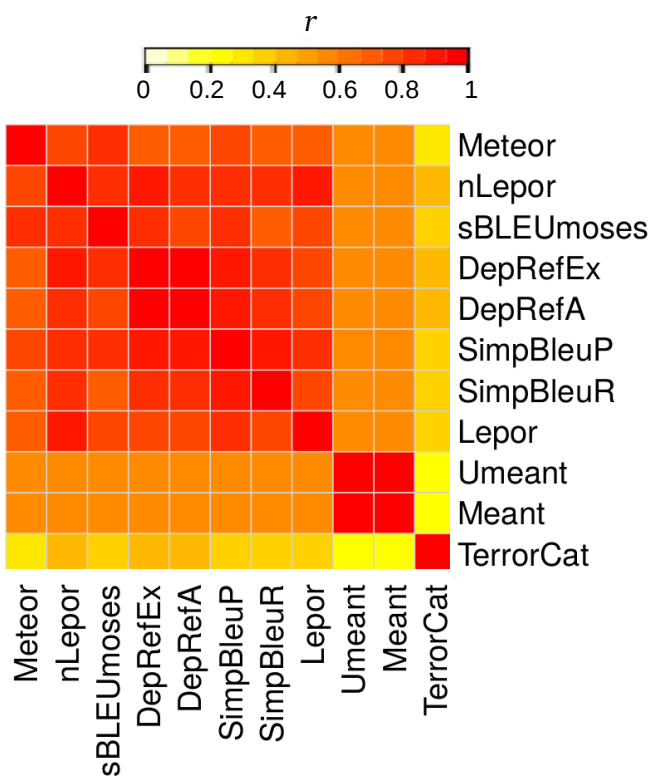

Figure 3: Pearson's correlation between every pair of segment-level metric competing in the WMT-13 Spanishto-English task.

the new assessment reflects how well metrics score translations of very close or equal quality, and, as described in Section 2, ameliorates the issue of low inter-annotator agreement as well as resolving the original mismatch between discrete human relative preference judgments and continuous metric scores.

Figure 3 is a heat map of the Pearson's correlation between each pair of segment-level metrics for Spanish-to-English from WMT-13, and Figure 4 shows correspondence between scores of three segment-level metrics with our human evaluation data. Figure 5 displays the outcome of the Williams significance test as applied to each pairing of competing metrics. Since the power of Williams test increases with the strength of correlation between a pair of metrics, it is important not to conclude the best system by the number of other metrics it outperforms. Instead, the best choice of metric for that language pair is any metric that is not signicifantly outperformed by any other metric. Three metrics prove not to be significantly outperformed by any other metric for Spanish-to-English, and tie for best performance: METEOR (Denkowski and Lavie, 2011), NLEPOR (Han et al., 2013) and SENTBLEU-MOSES (sBLEU-moses).
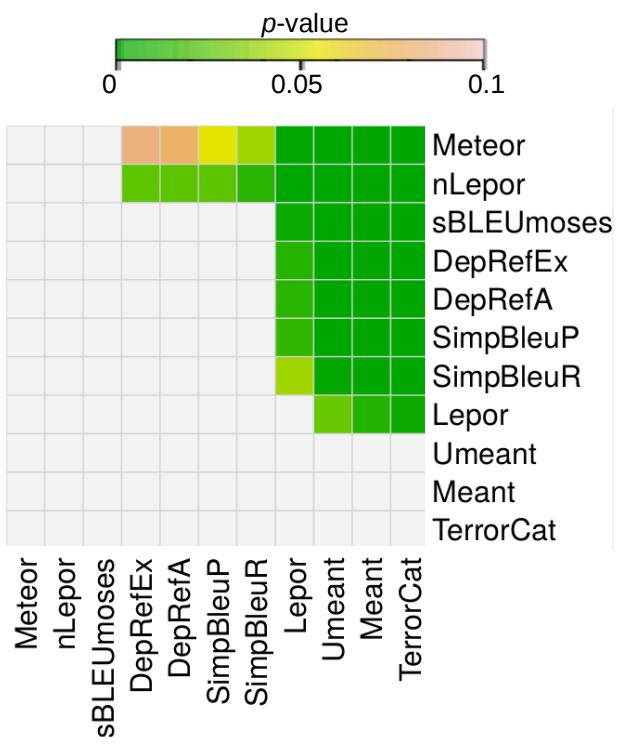

Figure 5: Evaluation of significance of increase in correlation with human judgment between every pair of segment-level metrics competing in the Spanish-toEnglish WMT-13 metrics task. A colored cell $(i, j)$ indicates that system named in row $i$ significantly outperforms system named in column $j$ at $p<0.1$, and green cells at $p<0.05$.

\begin{tabular}{lc}
\hline Metric & $r$ \\
\hline METEOR & 0.441 \\
NLEPOR & 0.416 \\
SENTBLEU-MOSES & 0.422 \\
SIMPBLEUP & 0.418 \\
SIMPBLEUR & 0.404 \\
LEPOR & 0.326 \\
\hline
\end{tabular}

Table 3: Pearson's correlation between each WMT-13 segment-level metric and human assessment for the combined set of nine language pairs.

\subsection{Language Pairs}

Since human assessments are now absolute, scores effectively have the same meaning across language pairs, facilitating the combination of data across multiple language pairs. Since many approaches to MT are language-pair independent, the ability to know what segment-level metric works best across all language pairs is useful for choosing an appropriate default metric or simply avoiding having to swap and change metrics across different language 

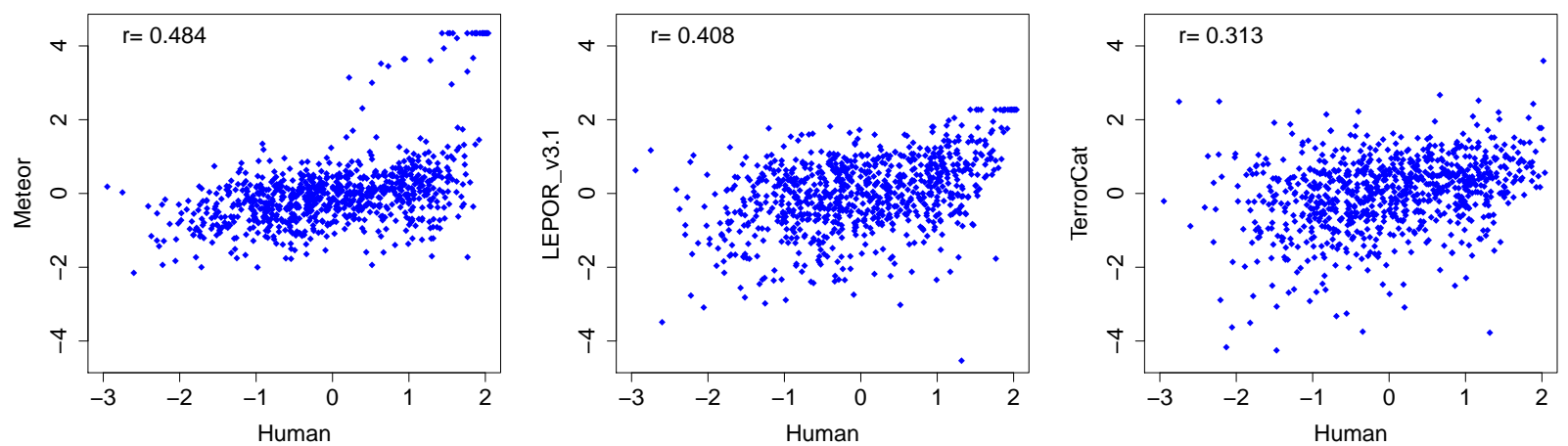

Figure 4: Standardized segment-level scores for human vs. metric over the WMT-13 Spanish-to-English segment-level metric task, for a metric achieving highest, mid-range and lowest Pearson's correlation with human judgment.

pairs.

Assessments of translations were crowd-sourced for nine language pairs used in the WMT-13 shared metrics task: Russian-to-English, Spanish-toEnglish, French-to-English, German-to-English, Czech-to-English, English-to-Russian, Englishto-Spanish, English-to-French and English-toGerman. ${ }^{4}$ Again, we obtain a minimum of 15 assessments per translation, and collect scores for 100 translations per language pair. After removal of quality control items, this leaves 70 distinct translations per language pair, combined into a cross-lingual test set of 630 distinct translations spanning nine language pairs.

Table 3 shows Pearson's correlation with human assessment for the six segment-level metrics that competed across all language pairs in WMT-13, and Figure 6 shows the outcomes of Williams test for statistical significance between different pairings of metrics. Results reveal that the same three metrics as before (METEOR, SENTBLEU-MOSES and NLEPOR), in addition to SIMPBLEUP and SIMPBLEUR are not significantly outperformed by any other metric at $p<0.05$. However, since the latter two were shown to be outperformed for Spanish-to-English, all else being equal, METEOR, SENTBLEU-MOSES and NLEPOR are still a superior choice of default metric.

\footnotetext{
${ }^{4}$ We were regrettably unable to include English-to-Czech, due to a lack of Czech-speaking MTurk workers.
}

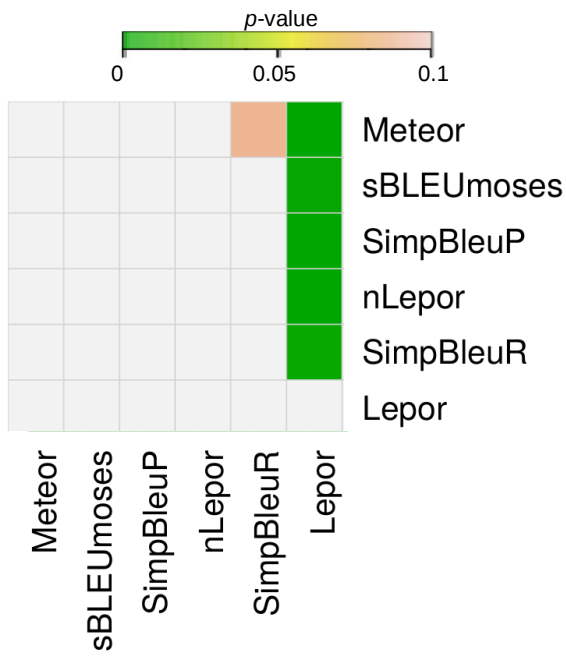

Figure 6: Evaluation of significance of increase in correlation with human judgment between every pair of segment-level metrics competing in all nine in WMT-13 metrics task. A colored cell $(i, j)$ indicates that system named in row $i$ significantly outperforms system named in column $j$ at $p<0.1$ and green cells specifically $p<0.05$.

\section{Conclusion}

We presented a new evaluation methodology for segment-level metrics that overcomes the issue of low inter-annotator agreement levels in human assessments, includes evaluation of very close and equal quality translations, and provides a significance test that supports system comparison with confidence. Our large-scale human evaluation reveals three metrics to not be significantly outperformed by any other metric in both Spanish-to- 
English and a combined evaluation across nine language pairs, namely: METEOR, NLEPOR and SENTBLEU-MOSES.

\section{Acknowledgements}

We wish to thank the anonymous reviewers for their valuable comments. This research was supported by funding from the Australian Research Council and Science Foundation Ireland (Grant 12/CE/12267).

\section{References}

W. Aziz, S. Castilho, and L. Specia. 2012. PET: a tool for post-editing and assessing machine translation. In Proc. of the 8th International Conference on Language Resources and Evaluation (LREC 2012), pages 39823987, Istanbul, Turkey.

O. Bojar, C. Buck, C. Callison-Burch, C. Federmann, B. Haddow, P. Koehn, C. Monz, M. Post, R. Soricut, and L. Specia. 2013a. Findings of the 2013 Workshop on Statistical Machine Translation. In Proceedings of the Eighth Workshop on Statistical Machine Translation, pages 1-44, Sofia, Bulgaria.

O. Bojar, C. Buck, C. Callison-Burch, C. Federmann, B. Haddow, P. Koehn, C. Monz, M. Post, R. Soricut, and L. Specia. 2013b. Findings of the 2013 Workshop on Statistical Machine Translation. In Proc. 8th Wkshp. Statistical Machine Translation, pages 1-44, Sofia, Bulgaria.

O. Bojar, C. Buck, C. Federmann, B. Haddow, P. Koehn, J. Leveling, C. Monz, P. Pecina, M. Post, H. SaintAmand, R. Soricut, L. Specia, and A. Tamchyna. 2014. Findings of the 2014 Workshop on Statistical Machine Translation. In Proc. 9th Wkshp. Statistical Machine Translation, pages 12-58, Baltimore, USA.

C. Callison-Burch, C. Fordyce, P. Koehn, C. Monz, and J. Schroeder. 2007. (Meta-) evaluation of machine translation. In Proc. 2nd Wkshp. Statistical Machine Translation, pages 136-158, Prague, Czech Republic.

C. Callison-Burch, C. Fordyce, P. Koehn, C. Monz, and J. Schroeder. 2008. Further meta-evaluation of machine translation. In Proc. 3rd Wkshp. Statistical Machine Translation, pages 70-106, Columbus, USA.

C. Callison-Burch, P. Koehn, C. Monz, and J. Schroeder. 2009. Findings of the 2009 Workshop on Statistical Machine Translation. In Proc. 4th Wkshp. Statistical Machine Translation, pages 1-28, Athens, Greece.

M. Denkowski and A. Lavie. 2011. Meteor 1.3: Automatic metric for reliable optimization and evaluation of machine translation systems. In Proc. 6th Wkshp. Statistical Machine Translation, pages 85-91, Edinburgh, Scotland.
Y. Graham and T. Baldwin. 2014. Testing for significance of increased correlation with human judgment. In Proceedings of the Conference on Empirical Methods in Natural Language Processing, pages 172-176, Doha, Qatar.

Y. Graham, T. Baldwin, A. Moffat, and J. Zobel. 2013. Continuous measurement scales in human evaluation of machine translation. In Proc. 7th Linguistic Annotation Wkshp. \& Interoperability with Discourse, pages 33-41, Sofia, Bulgaria.

Y. Graham, T. Baldwin, A. Moffat, and J. Zobel. 2014a. Is machine translation getting better over time? In Proceedings of the European Chapter of the Association of Computational Linguistics, pages 443-451, Gothenburg, Sweden.

Y. Graham, N. Mathur, and T. Baldwin. 2014b. Randomized significance tests in machine translation. In Proc. Ninth ACL Wkshp. Statistical Machine Translation, pages 266-74, Baltimore, MD.

A.L. Han, D.F. Wong, L.S. Chao, Y. Lu, L. He, Y. Wang, and J. Zhou. 2013. A description of tunable machine translation evaluation systems in WMT13 metrics task. In Proceedings of the Eight Workshop on Statistical Machine Translation, pages 414-421, Sofia, Bulgaria.

P. Koehn. 2004. Statistical significance tests for machine translation evaluation. In Proc. of Empirical Methods in Natural Language Processing, pages 388-395, Barcelona, Spain.

M. Machacek and O. Bojar. 2014. Results of the wmt14 metrics shared task. In Proceedings of the Ninth Workshop on Statistical Machine Translation, pages 293301, Baltimore, USA.

J.H. Steiger. 1980. Tests for comparing elements of a correlation matrix. Psychological Bulletin, 87(2):245.

E.J. Williams. 1959. Regression analysis, volume 14. Wiley, New York, USA. 